\title{
INJECTION CHOICE FOR SPALLATION NEUTRON SOURCE RING*
}

\author{
J. Wei ${ }^{\dagger}$, J. Beebe-Wang, M. Blaskiewicz, J. Brodowski, A. Fedotov, C. Gardner, Y. Y. Lee, \\ D. Raparia, BNL, USA; V. Danilov, J. Holmes, ORNL, USA \\ C. Prior, G. Rees, RAL, United Kingdom; S. Machida, KEK, Japan
}

\begin{abstract}
Injection is key in the low-loss design of high-intensity proton facilities like the Spallation Neutron Source (SNS). During the design of both the accumulator and the rapidcycling-synchrotron version of the SNS, extensive comparison has been made to select injection scenarios that satisfy SNS's low-loss design criteria. This paper presents issues and considerations pertaining to the final choice of the SNS injection systems.
\end{abstract}

\section{INTRODUCTION}

The SNS accumulator ring is designed to compress proton beams at $1 \mathrm{GeV}$ kinetic energy and deliver to the target a beam power of $2 \mathrm{MW}$. The charge-exchange system accepts 1060 turns of chopped $\mathrm{H}^{-}$beam and paints both the transverse and longitudinal phase space. The design goal is an operationally robust injection system, independently flexible for both transverse and longitudinal manipulations [1] to alleviate space-charge effects and instabilities, and to satisfy the target requirements on beam distribution.

\section{LATTICE AND LAYOUT}

The ring lattice is designed with a dispersion-free region consisting of three long drifts using two pairs of quadrupole doublets. As shown in Fig. 1, the center drift of $12.5 \mathrm{~m}$ length houses the entire injection chicane, allowing robust lattice tuning without affecting the trajectories of the injecting $\mathrm{H}^{-}$and $\mathrm{H}^{0}$ particles. The two side drifts, each of $6.85 \mathrm{~m}$ length, symmetrically house dynamic kickers for rising and falling orbit bumps for horizontal $(\mathrm{H})$ and vertical $(\mathrm{V})$ painting.

Injecting in a zero-dispersion region makes possible independent control of painting in all three directions. Table 1 compares the features of zero-dispersion and highdispersion injection schemes [2]. Instead of collection upon injection, any possible momentum tail of the incoming beam is cleaned either with $\mathrm{H}^{-}$collimators before injection, or with the a beam-in-gap kicker inside the ring. The zero-dispersion region also allows possible future novel schemes like laser-stripping [3] injection.

\section{TRANSVERSE PAINTING}

Transverse painting alleviates the fundamental spacecharge limit and controls the uniformity and shape of the beam profile. Various beam profiles can be achieved using fast orbit bump or injection steering. The injection system is designed to facilitate painting in both the horizontal

\footnotetext{
${ }^{*}$ Work performed under the auspices of the US Department of Energy
}

$\dagger$ wei1@bnl.gov, on joint appointment at ORNL and BNL

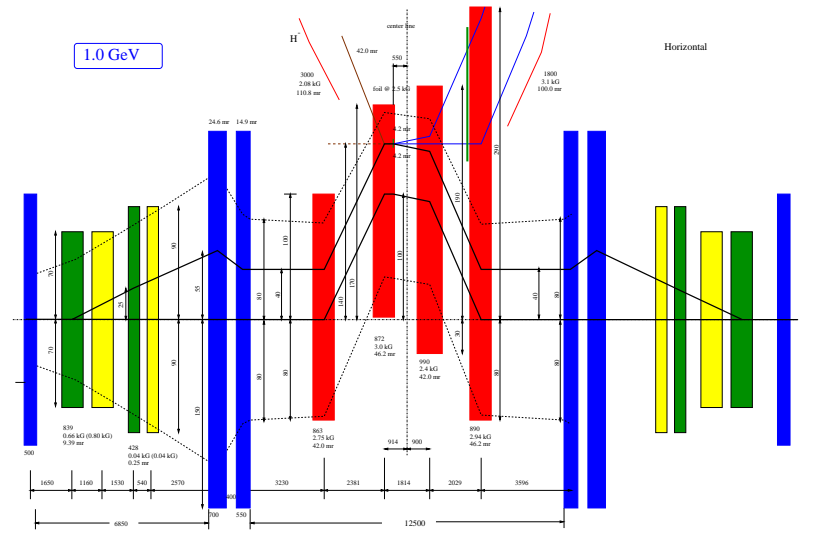

Figure 1: SNS dispersion-free injection. Elements shown are the chicane (red), the ring lattice quadrupoles (blue), and dynamic kickers (yellow $\mathrm{H}$ and green $\mathrm{V}$ ).

Table 1: Comparison of zero-dispersion and highdispersion injection schemes.

\begin{tabular}{ll}
\hline High-Dispersion & Zero-Dispersion \\
\hline Long, low-field lattice dipole & Dipole chicane \\
No injecting septum & Needs injecting septum \\
No horizontal kickers & Needs horizontal kickers \\
Input energy tail collection & Beam-in-gap cleaning \\
Tight input $\Delta p / p$ & Relaxed input $\Delta p / p$ \\
Coupled H-L painting & Independent $\mathrm{H}, \mathrm{V}, \mathrm{L}$ adjust \\
\hline
\end{tabular}

and vertical planes with either correlated or anti-correlated bumps [4]. Hybrid schemes utilizing orbit bumps in one direction and injection-angle steering in the other were debated but not adopted.

\subsection{Correlated-Bump Painting}

Correlated painting (Fig. 2) has the advantage that the beam halo is constantly painted over by freshly injected beam. The main concern is whether the rectangular beam profile is preserved, or evolves in a controlled way, in the

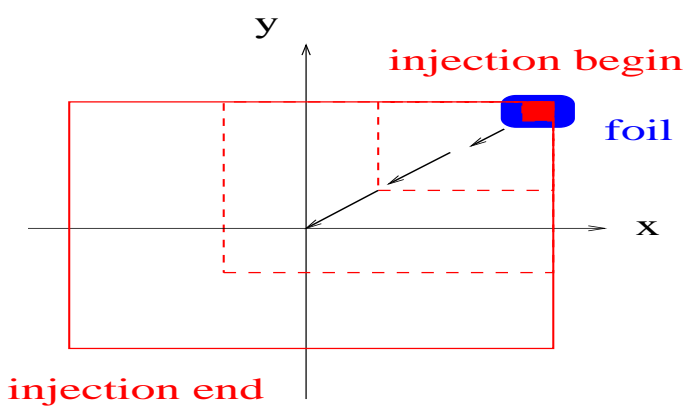

Figure 2: Transverse profile of correlated painting. 

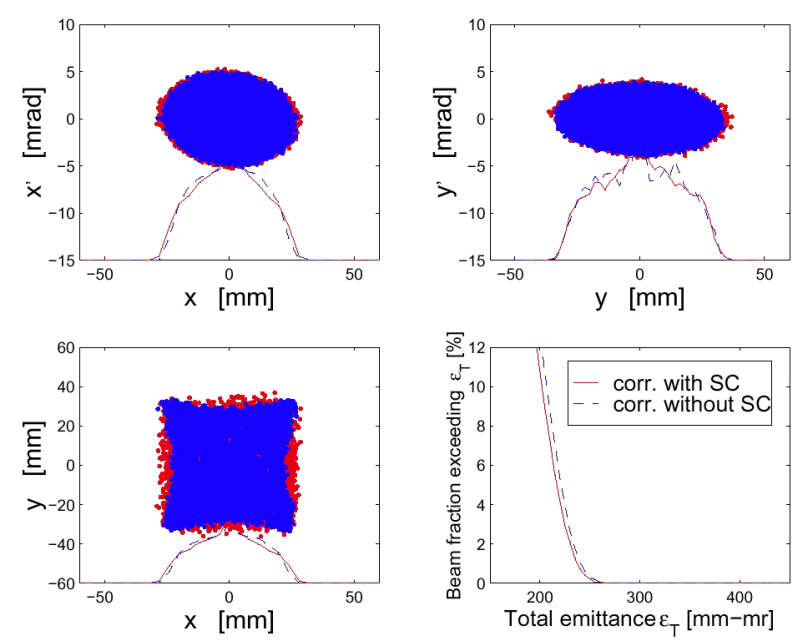

Figure 3: Rectangular transverse beam profile resulting from correlated painting. Space charge produces an insignificant beam tail (shown in red).

presence of coupling produced by space charge and magnet errors. Space charge smears the diagonal density singularity in $x-y$ space.

\subsection{Anti-Correlated-Bump Painting}

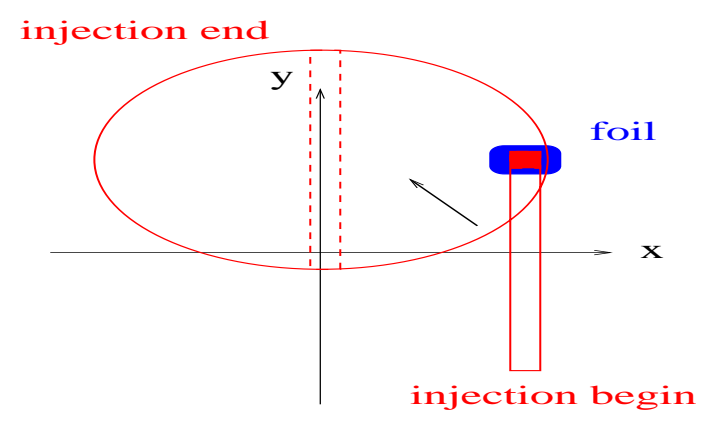

Figure 4: Transverse profile of anti-correlated painting.

Anti-correlated painting utilizes both horizontal and vertical orbit bumps, one programmed with increasing and the other with decreasing amplitude. Ideally, this produces a distribution with an elliptical transverse profile and a uniform density distribution. In the presence of space charge, significant beam halo is produced during the early stage of painting, when the beam is narrow in one direction. Also, approximately $50 \%$ extra aperture is needed in the direction of large starting bump amplitude.

\subsection{Painging/Steering Hybrid}

A uniform distribution can also be realized by painting in one direction and steering in the other, as shown in Fig. 5. The four vertical kickers in the ring are replaced by a small vertical kicker in the transport line at a betatron phase of $\pi$ upstream of the injection septum to vary the injection beam angle. With such a scheme, a challenging issue is that the foil needs to be supported horizontally to avoid excessive foil traversal and scattering. An alternative scheme

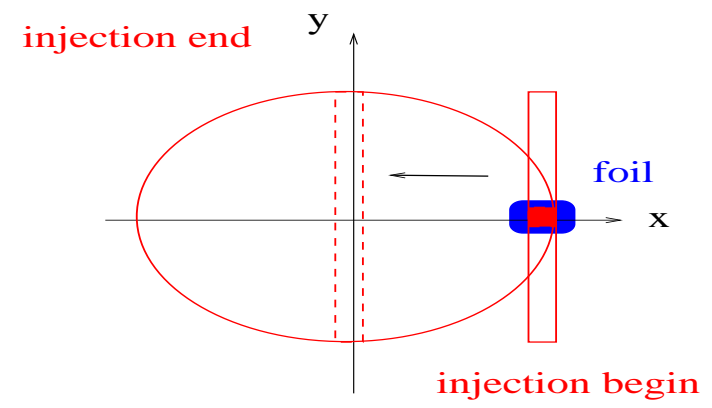

Figure 5: Transverse profile of $\mathrm{H}$ painting/V steering.

Table 2: Comparison of transverse painting schemes for zero-dispersion injection.

\begin{tabular}{cll}
\hline Type & Advantage & Disadvantage \\
\hline Correlated & $\begin{array}{l}\text { Paint over halo } \\
\text { (Square profile) }\end{array}$ & $\begin{array}{l}\text { Singular density } \\
\text { Coupling growth }\end{array}$ \\
Anti- & $\begin{array}{l}\text { Immune to coupling Halo growth } \\
\text { correlated }\end{array}$ & $\begin{array}{l}\text { (Circular profile) } \\
\text { Extra aperture }\end{array}$ \\
H-V & Paint over halo & Extra aperture \\
coupled & (Diamond profile) & \\
Paint (H)/ & similar to anti-corr. & Foil support difficult \\
steer (V) & fewer kicker & Suscep. operation error \\
Paint (V) & similar to anti-corr. & Vertical injection \\
/steer (H) & fewer kicker & Suscep. operation error \\
Oscil. bump & Paint over halo & Fast PS switch \\
& (Circular profile) & Extra aperture (H\&V) \\
\hline
\end{tabular}

is to use vertical orbit painting and horizontal angle steering. However, the beam needs to be injected vertically. In both cases, the acceptance of the injection channel all the way up to the injection dump needs to accommodate the varying injection beam angle. Operationally, the reliability of the kicker system upstream of injection must be high to avoid injection foil miss, dump over-heating, and injection channel activation.

\subsection{Other Schemes}

By oscillating the $\mathrm{H}$ and $\mathrm{V}$ bump, a $(X-Y)$ circular beam profile may be realized without much halo growth. However, the required power supply switching is difficult, and extra aperture is needed in both $\mathrm{H}$ and $\mathrm{V}$ directions. Intentional transverse coupling may also alter the painted beam profile.

\section{LONGITUDINAL PAINTING}

Longitudinal painting provides the momentum spread required for beam stability without introducing excessive momentum halo. With dispersion-free injection, longitudinal painting can be achieved for linac-to-ring injection by using an "energy spreader" RF cavity located in the transport line upstream of the injection region, operating at a phase-modulated mode of the linac frequency. In order to facilitate such a painting scheme, the output momentum jitter and spread need to be strictly controlled by an "energy corrector" RF cavity synchronized to linac frequency at an 


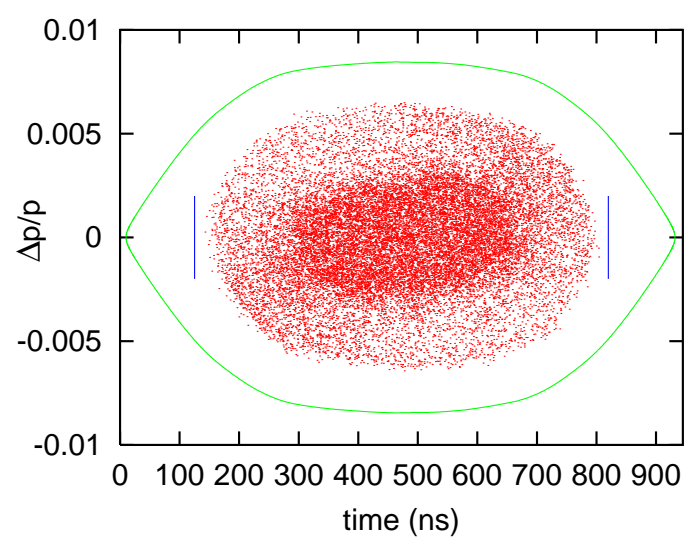

Figure 6: Longitudinal phase space at the end of 2 MW beam accumulation. The effects of space charge and cavity beam loading are included.

optimized distance from the end of linac to allow for an adequate beam-phase slippage and thus a moderate RF voltage.

\section{DESIGN CONSIDERATIONS}

\subsection{Energy Acceptance}

The magnetic field inside and upstream of the stripping field is designed below $3 \mathrm{kG}$ to maintain an $\mathrm{H}^{-}$magnetic stripping loss below $10^{-8}$ per meter. The field of the second and third (middle two) chicane dipoles are 3 and 2.4 $\mathrm{kG}$, while the foil sits in the falling field of the second dipole at $2.5 \mathrm{kG}$, allowing $\mathrm{H}^{0}$ of Stark state $n \geq 5$ to be immediately stripped, while those of $n \leq 4$ stripped only by the second foil. The energy acceptance of the injecting beam is $\pm 5 \%$, determined by allowing less than about $10^{-5}$ loss at injection. In the case of an upgraded energy from 1 to $1.3 \mathrm{GeV}$, the second and third chicane dipoles are replaced by magnets of $40 \%$ longer length (L) (field $B \propto(\beta \gamma)^{-1}$ for stripping, $\left.B L \propto \beta \gamma, L \propto(\beta \gamma)^{2}\right)$.

\subsection{Optics Perturbation}

For the nominal transverse tunes of $(6.4,6.3)$, the maximum amplitude distortion $(\Delta \beta / \beta)$ is $5 \%$ from both fixed chicane and dynamic bump. The maximum residual dispersion is $0.1 \mathrm{~m}$ from the fixed chicane and $0.25 \mathrm{~m}$ from both the chicane and bump. As the tune varies [5], the change of $\beta_{x, y}$ at the foil location can be as much as $50 \%$. Extra aperture is reserved, and the bump amplitude is adjusted accordingly for a specific painting emittance.

\subsection{Aperture Requirements}

The injection admittance is designed to be $480 \pi \mu \mathrm{r}$ to accommodate a beam of unnormalized emittance $240 \pi \mu \mathrm{r}$. The collimator admittance is at $300 \pi \mu \mathrm{r}$. Extra aperture is reserved for tune adjustment (50\% $\mathrm{H}$ and $\mathrm{V}$ emittance), optional anti-correlated painting (50\% V emittance), and the extra orbit bump to move the beam away from foil upon the completion of injection.

\subsection{Foil Heating and Exchange}

Foil width is minimized by mismatching [6] the injection beam Courant-Snyder parameters $\left(\beta_{i}, \alpha_{i}\right)$ from the ring value $(\beta, \alpha)$ by

$$
\frac{\beta_{i}}{\beta}=\frac{\alpha_{i}}{\alpha}=\left(\frac{\epsilon_{i}}{\epsilon}\right)^{1 / 3}
$$

where the 99\% unnormalized emittance is $\epsilon_{i}=2.5 \pi \mu \mathrm{r}$ for the injecting beam, and $\epsilon \approx 240 \pi \mu \mathrm{r}$ for the painted beam. During a 1060-turn injection, the average number of foil traversals is 6 for correlated and 8 for anti-correlated painting. The foil is planned to be positioned at an angle to increase its effective thickness. An exchange system is designed to accommodate up to 32 foils.

\subsection{Magnet Field Error Tolerance}

The tolerable fractional field error is $0.1 \%$ for the chicane dipoles, and $1 \%$ for the dynamic kickers respectively, integrated at full vacuum chamber acceptance. Requirements on the magnet power supplies are similar. For comparison, the tolerable field and power supply error is $0.01 \%$ for the main lattice dipoles and quadrupoles.

\subsection{Electron Collection}

Electrons stripped by the foil are guided by the end field of the second chicane dipole, with specially tapered pole tip [7], and collected by a water-cooled $\mathrm{Cu}$ block located at the bottom of the chamber. The pole tip of the third chicane dipole is also tapered accordingly to compensate the field deviation.

\subsection{Injection Dump}

Any $\mathrm{H}^{-}$and $\mathrm{H}^{0}$ beam survived and produced by the first foil is stripped by the second foil and then focused to the injection dump. The dump is designed to accept up to $10 \%$ $(200 \mathrm{~W})$ of the peak power.

\section{SUMMARY}

SNS chooses $\mathrm{H}^{-}$injection in a zero-dispersion region allowing independent control of horizontal, vertical and longitudinal phase-space painting. Nominally operating at 1 $\mathrm{GeV}$, the system is designed for straightforward upgrade to $1.3 \mathrm{GeV}$ with replacement of two chicane dipoles.

\section{REFERENCES}

[1] J. Wei et al, Phys. Rev. ST-AB 3, 080101 (2000).

[2] G. Rees, EPAC 1994, p.241; G. Rees, in Handbook of Accelerator Physics and Engineering, ed. A. Chao, M. Tigner, World Scientific (1998) p.497.

[3] Y. Suzuki, I. Yamane, Workshop on Injection and Extraction, Abingdon (1999).

[4] J. Beebe-Wang et al, EPAC 2000, p.1465; J. Galambos, et al, Phys. Rev. ST-AB, 3, 034201 (2000).

[5] J. Holmes, et al, these proceedings.

[6] C. Prior, G. Rees, 12th HIF sym. (1997).

[7] D. Abell et al, EPAC 2000, p.2107. 